\title{
gु \\ Electrodynamic response and local tunneling spectroscopy of strongly disordered superconducting TiN films
}

\author{
P. C. J. J. Coumou, ${ }^{1, *}$ E. F. C. Driessen, ${ }^{2, \dagger}$ J. Bueno, ${ }^{3}$ C. Chapelier, ${ }^{2}$ and T. M. Klapwijk ${ }^{1,4}$ \\ ${ }^{1}$ Kavli Institute of Nanoscience, Delft University of Technology, Delft, The Netherlands \\ ${ }^{2}$ SPSMS/LATEQS, UMR-E 9001, CEA-INAC and Université Joseph Fourier, Grenoble, France \\ ${ }^{3}$ SRON Netherlands Institute for Space Research, Utrecht, The Netherlands \\ ${ }^{4}$ Physics Department, Moscow State Pedagogical University, 119991 Moscow, Russia
}

(Received 10 October 2013; published 7 November 2013)

\begin{abstract}
We have studied the electrodynamic response of strongly disordered superconducting TiN films using microwave resonators, where the disordered superconductor is the resonating element in a high-quality superconducting environment of NbTiN. We describe the response assuming an effective pair-breaking mechanism modifying the density of states and compare this to local tunneling spectra obtained using scanning tunneling spectroscopy. For the least disordered film $\left(k_{\mathrm{F}} l=8.7, R_{\mathrm{s}}=13 \Omega\right)$, we find good agreement, whereas for the most disordered film $\left(k_{\mathrm{F}} l=0.82, R_{\mathrm{s}}=4.3 \mathrm{k} \Omega\right.$ ), there is a strong discrepancy, which signals the breakdown of a model based on uniform properties.
\end{abstract}

DOI: 10.1103/PhysRevB.88.180505

PACS number(s): 74.81.-g, 74.25.N-, 74.62.En, 74.55.+v

The evolution of the superconducting state with increasing disorder is an important and complex problem. Many processes are present simultaneously, and the predominant experimental outcome is that the system evolves towards an insulating state ${ }^{1}$ (i.e., increasing resistance with decreasing temperature) while, at the same time, maintaining properties reminiscent of Cooper pairs. ${ }^{2}$ Recently, we have carried out ${ }^{3}$ a systematic study of the microwave response of TiN films with values of the Ioffe-Regel parameter $k_{\mathrm{F}} l$ ranging from 2.4 to 8.6. These results have been compared with a recent theory of Feigel'man and Skvortsov, ${ }^{4}$ designed to describe the effect of disorder and the emergence of mesoscopic inhomogeneities on the quasiparticle density of states in the superconducting state. It was found that the effects of disorder become visible already at a much lower level than was predicted by this theory. To analyze the results, we have therefore used a heuristic model which treats the effect of disorder as a pair-breaking parameter that is not a priori related to the level of disorder. Here, we extend the experimental results to films with sheet resistance $R_{\mathrm{S}}$ up to $4.3 \mathrm{k} \Omega$ and $k_{\mathrm{F}} l$ values down to 0.82 . We observe a trend similar to that reported previously, with a strong increase in the effective pair-breaking parameter for decreasing $k_{\mathrm{F}} l$. Since the analysis assumes a modified density of states we supplement our analysis with data derived from local tunneling spectroscopy on the same films. For the least disordered film $\left(k_{\mathrm{F}} l=8.6, R_{\mathrm{s}}=13 \Omega\right)$, we find good agreement, but for the most disordered film $\left(k_{\mathrm{F}} l=\right.$ $0.82, R_{\mathrm{s}}=4.3 \mathrm{k} \Omega$ ), there is a strong discrepancy between the locally measured density of states and the density of states that has been assumed to describe the electrodynamic response.

To measure the electrodynamics of superconductors, commonly coplanar waveguide resonators are used, which are capacitively coupled to a readout transmission line, where the resonator, the ground plane, and the transmission line are all made of the same material (i.e., the superconductor under study). In the regime where $k_{\mathrm{F}} l \approx 1$, this approach becomes unfeasible. The high sheet resistance in this regime $\left(R_{\square}>1 \mathrm{k} \Omega\right.$ ) translates into an equally high sheet inductance $L_{\mathrm{S}} \approx \hbar R_{\square} / \pi \Delta$, with $\Delta$ the superconducting pairing potential. For increasing disorder, it therefore becomes increasingly difficult to match the characteristic impedance of the transmission line $Z \approx \sqrt{L / C}$ to the $50-\Omega$ impedance of the microwave equipment, with $L$ and $C$ the total inductance and capacitance, respectively. This results in standing waves in the transmission line and spurious resonances.

To address this issue, we have adapted a resonator design by Diener et al. ${ }^{5}$ In our design, a half-wave coplanar waveguide resonator of TiN is embedded in an environment of 100-nmthick NbTiN. First, the resonator structure is defined in the TiN film, using e-beam lithography and reactive ion etching. Second, the NbTiN layer is deposited on a liftoff mask, using dc magnetron sputtering, at a dc power of $300 \mathrm{~W}$, using an argon flow of $100 \mathrm{sccm}$ and a nitrogen flow of $4 \mathrm{sccm}$, with a chamber pressure of 4 mTorr. ${ }^{6}$ Liftoff of the NbTiN is done in acetone. ${ }^{7}$ Figure 1(a) shows an optical micrograph of one of the resonators.

Stronger disorder also results in a lower internal quality factor $Q_{\mathrm{i}}$ of the resonator. ${ }^{8}$ Changes in the properties of the resonators are best assessed when $Q_{\mathrm{i}} \approx\left|Q_{\mathrm{c}}\right|$, with $Q_{\mathrm{c}}$ the complex quality factor corresponding to the (dominantly capacitive) coupling to the transmission line. ${ }^{9}$ Maximal coupling (minimal $\left|Q_{\mathrm{c}}\right|$ ) is obtained by positioning the resonators such that half of their length is adjacent to the transmission line. This design yields a coupling quality factor of $\left|Q_{\mathrm{c}}\right| \approx 3 \times 10^{3}$, which should be compared to the typical $Q_{\mathrm{i}} \leqslant 10^{4}$. Detailed dimensions of the sample design are given in the Supplemental Material. ${ }^{10}$ Figure 1(b) shows a typical resonance curve of a resonator made from film $\mathrm{E}$ using this design. The resonance dip is clearly visible and can be followed up to a temperature of $417 \mathrm{mK}$.

The TiN films used in this study are grown using plasmaassisted atomic-layer deposition (ALD). The ALD technique is based on sequential self-terminating gas-solid reactions at the film surface, using two gaseous precursors. We make use of the precursors $\mathrm{TiCl}_{4}$ and a plasma of $\mathrm{H}_{2}$ and $\mathrm{N}_{2}$, which react into TiN and gaseous $\mathrm{HCl}$. The films are deposited on highly resistive $(\rho>10 \mathrm{k} \Omega \mathrm{cm}) \mathrm{Si}(100)$ substrates that have a thin surface layer of native silicon oxide. The level of disorder is tuned by changing the thickness $d$ of the films $s^{3,8}$ 

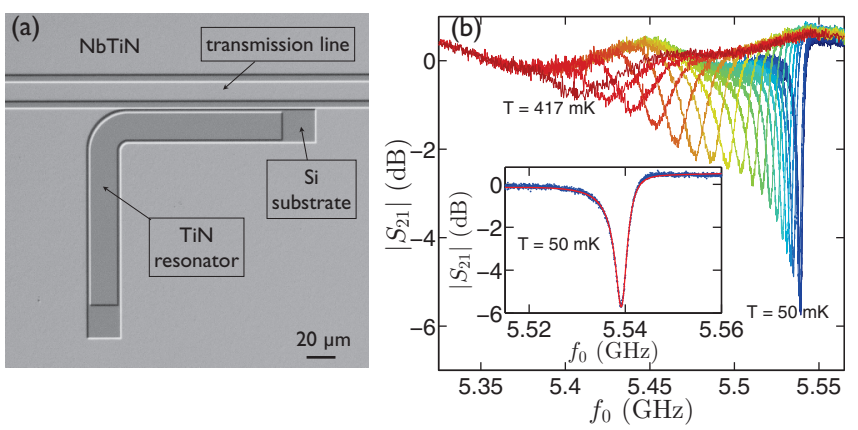

FIG. 1. (Color online) (a) Optical microscope image of the resonator design used in this paper. A TiN half-wave resonator is embedded in a NbTiN ground plane and capacitively coupled to the NbTiN transmission line. (b) Transmission line throughput $S_{21}$ as a function of microwave frequency, for a resonator from film $\mathrm{E}$. The design allows us to follow the resonance curve for a temperature range from $T=50 \mathrm{mK}$ (blue curve) to $T=417 \mathrm{mK}$ (red curve). Inset: The curve for $T=50 \mathrm{mK}$ (circles) together with a fit of Eq. (1) to the measured data [solid (red) curve].

(see Table I, films C, E, and G). The resulting films are not directly comparable to the previously studied films (notably, the critical temperature is slightly higher), due to a maintenance update of the machine. ${ }^{11}$ The thick film I is part of the previous deposition series. $^{12}$

Our ALD process yields thin films with a typical RMS surface roughness of $2 \AA$, determined by atomic force microscopy. Cross-sectional transmission electron microscopy of film $\mathrm{C}$ reveals that the TiN layer is polycrystalline, with a typical grain size of $5 \mathrm{~nm}$. The thickness of the various films is estimated using an average growth rate of $0.5 \AA /$ cycle. $^{10}$

Hall bar structures patterned in each film are used to measure the sheet resistance as a function of temperature $T$ and the Hall voltage $V_{\mathrm{H}}(B)$ as a function of magnetic field. The critical temperature $T_{\mathrm{c}}$ is determined from the the resistive transition. ${ }^{10}$ For our films, the superconductor-to-insulator transition (SIT) occurs at a sheet resistance between 4.3 and $16 \mathrm{k} \Omega$, consistent with previously reported values for the SIT in TiN. ${ }^{13}$ The sheet resistance, elastic scattering length $l$, and $k_{\mathrm{F}} l$ are determined at a temperature of $10 \mathrm{~K}$, using free-electron theory. ${ }^{10}$ The Ioffe-Regel parameter $k_{\mathrm{F}} l$ decreases monotonously with film thickness, to values $<1$ for film $\mathrm{C}$, indicating strong electron localization. Films $\mathrm{C}-\mathrm{G}$ are part of a larger series, described in the Supplemental Material. $^{10}$

We have fabricated resonator samples from these films, using the design described before. The samples are wire

TABLE I. Parameters of the films. The values of $\Delta_{0}$ and $\alpha$ are extracted from the fits to the measurements of the electrodynamics.

\begin{tabular}{lccccccc}
\hline \hline Film & $\begin{array}{c}\text { Thickness } \\
(\mathrm{nm})\end{array}$ & $\begin{array}{c}T_{\mathrm{c}} \\
(\mathrm{K})\end{array}$ & $\begin{array}{c}R_{s} \\
(\mathrm{k} \Omega)\end{array}$ & $\begin{array}{c}l \\
(\AA)\end{array}$ & $k_{F} l$ & $\begin{array}{c}\Delta_{0} \\
(\mu \mathrm{eV})\end{array}$ & $\alpha / \Delta_{0}$ \\
\hline $\mathrm{C}$ & 4.0 & 0.70 & 4.3 & 0.96 & 0.82 & 307 & 0.65 \\
$\mathrm{E}$ & 4.5 & 0.99 & 3.0 & 1.2 & 1.1 & 277 & 0.49 \\
$\mathrm{G}$ & 5.0 & 1.5 & 1.5 & 1.9 & 1.8 & 288 & 0.23 \\
$\mathrm{I}$ & 89 & 3.5 & 0.013 & 7.3 & 8.6 & 613 & 0.11 \\
\hline \hline
\end{tabular}

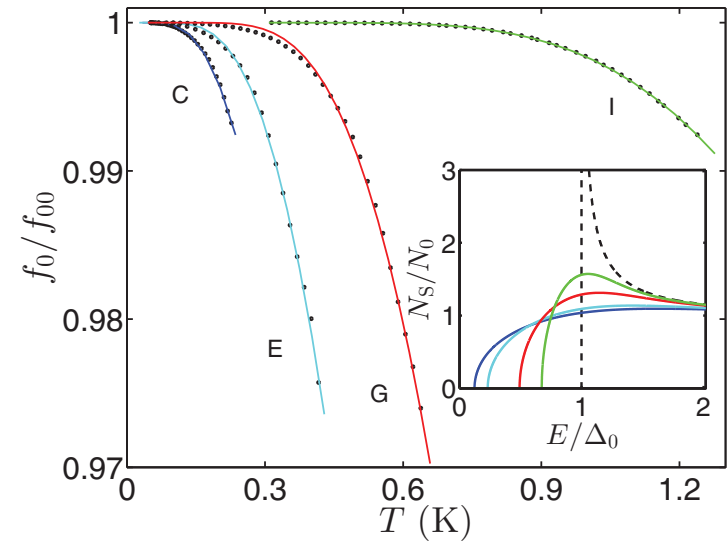

FIG. 2. (Color online) Measured resonance frequency as a function of temperature for films C, E, G, and I. Solid curves are a fit of the model to the data (see text for details). Inset: Superconducting densities of states corresponding to these fits, compared to the BCS density of states (dashed curve).

bonded to coaxial connectors of a cryogenic microwave setup and cooled to a base temperature of $50 \mathrm{mK}$ using a pulse-tube precooled adiabatic-demagnetization refrigerator. A microwave signal from a vector network analyzer is fed to the sample through coaxial cables. The amplified forward power transmission $S_{21}$ of the transmission line is recorded as a function of the temperature and microwave frequency [Fig. 1(b)]. A small linear background is observed in these measurements, due to small imperfections in the calibration of the setup. We subtract this background from the data and extract the resonance frequency $f_{0}$ by fitting the relation ${ }^{14}$

$$
\frac{1}{S_{21}}=1+\frac{Q_{\mathrm{i}} / Q_{\mathrm{c}}}{1+2 i Q_{\mathrm{i}} \frac{f-f_{0}}{f_{0}}},
$$

where $f$ is the microwave frequency. The solid curve in the inset in Fig. 1(b) is a fit of this line shape to the measured curve. Different resonators on the same chip show an identical temperature dependence. For increasing temperature, the resonance frequency $f_{0}$ is decreasing, reflecting a change in the sheet inductance $L_{S}$ of the superconductor. Figure 2 shows the change in resonance frequency as a function of temperature for all four measured films.

We model the microwave response using a description of the superconducting state, in which the superconductor is homogeneously weakened by the disorder-dependent pairbreaking parameter $\alpha$, similar to the effect of magnetic impurities. ${ }^{3}$ We assume homogeneous superconductivity and describe the superconducting state using the Usadel equation,

$$
i E \sin \theta+\Delta \cos \theta-\alpha \sin \theta \cos \theta=0,
$$

where $E$ is the quasiparticle energy, $\sin \theta$ and $\cos \theta$ are the quasiclassical Green's functions, and $\Delta$ is the pairing amplitude, which is determined self-consistently for each temperature and value of $\alpha{ }^{10}$ The effect of the pair-breaking parameter is to smoothen the coherence peak in the quasiparticle density of states, as shown in the inset in Fig. 2.

In contrast to our earlier work, here we take $\Delta_{0}$, the pairing amplitude at zero temperature, as a free parameter in the model. 
This choice is motivated by the observation that there is no $a$ priori relationship between $T_{\mathrm{c}}$ and $\Delta_{0}$ for this kind of film. ${ }^{15}$

After solving the Usadel equation, (2), the complex conductivity $\sigma=\sigma_{1}-i \sigma_{2}$ is determined using a generalized form of the Mattis-Bardeen equations, given by Nam. ${ }^{10,16}$ The resonance frequency $f_{0}$ is dependent only on $\sigma_{2}:{ }^{10}$

$$
f_{0} \propto \frac{1}{\sqrt{L_{\mathrm{S}}\left(\sigma_{2}\right)}} .
$$

The solid curves in Fig. 2 show the best fits of this model to the measured resonance frequencies. To obtain these fits, we use the resonance frequency at the lowest measured temperature, $f_{00}$, as a scaling parameter. For a number of values of $\alpha$, we determine a least-squares fit using $\Delta_{0}$ as the fitting parameter. Finally, we determine the value of $\alpha$ where the residual error of the fit was minimal. The resulting values of $\alpha$ and $\Delta_{0}$ are listed in Table I. As before, the trend that $\alpha$ increases with increasing disorder ${ }^{3}$ is also observed in these films. The inset in Fig. 2 shows the quasiparticle density of states $N_{\mathrm{S}} \propto \operatorname{Re} \cos \theta$, which corresponds to the fitted value of $\alpha$ for all four films.

Our model implies distinct predictions for the quasiparticle density of states of the film. To test these predictions, we independently probed the local density of states using scanning tunneling spectroscopy (STS) on unpatterned pieces of films $\mathrm{I}$ and $\mathrm{C}$. A piece of film I is mounted in a pumped $-{ }^{4} \mathrm{He}$ bath cryostat and cooled down to a bath temperature of $1.35 \mathrm{~K}$. Film $\mathrm{C}$ is mounted in an inverted ${ }^{3} \mathrm{He}-{ }^{4} \mathrm{He}$ dilution refrigerator and cooled down to a base temperature of $50 \mathrm{mK}$. A sharp Pt-Ir tip is brought in close proximity to the films, such that a tunneling current of $1 \mathrm{nA}$ is reached in both cases, at the maximum bias voltage of $2 \mathrm{mV}$ for film I and $500 \mu \mathrm{V}$ for film C. A low-frequency modulation with an amplitude of 10 and $20 \mu \mathrm{V}$, respectively, is applied to the dc voltage bias, to measure the differential conductance using lock-in techniques. Tunneling spectra are obtained by ramping the dc bias voltage, while keeping the distance between the tip and the sample fixed.

Figure 3(a) shows the tunneling spectrum on a single point of film I. The curve is an average of 50 consecutive spectra that were recorded at the same position. The spectrum shows the characteristic BCS coherence peaks at a voltage of $800 \mu \mathrm{V}$. A detailed scan over an area of $500 \times 500 \mathrm{~nm}^{2}$ shows that the variation in the local density of states is within the noise level of the measurement for this film.

A different picture is obtained from the measurements on film C. On this film, spectra were obtained on a square grid of $26 \times 26$ points in an area of $100 \times 100 \mathrm{~nm}^{2}$. Figure $3(\mathrm{~b})$ shows the average of the tunneling spectrum measured at all positions (solid curve). Clearly, the BCS coherence peaks are visible, although they are not as pronounced as in film I. Besides that, the tunneling conductivity increases with increasing voltage. This rise is also observed at temperatures above $T_{\mathrm{c}}$ and is generally attributed to a depression of the normal-state tunneling density of states at the Fermi level, caused by increased electron-electron interactions in the film. ${ }^{17,18}$ The dashed (red) curve in Fig. 3(b) shows the average background, measured over an area of $100 \times 100 \mathrm{~nm}^{2}$ at a temperature of $T=1.4 \mathrm{~K}$. The depression of the tunneling density of states is due to the energy cost of adding one electron to the film. Therefore, it is typical for tunneling and we do not expect
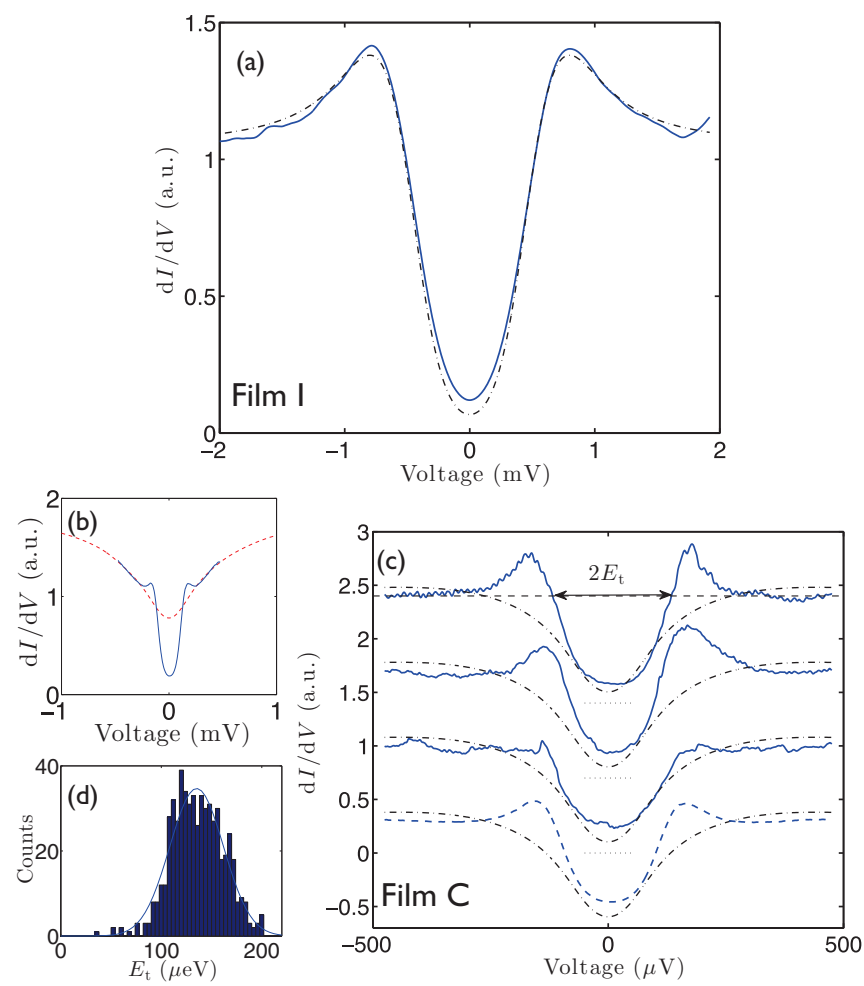

FIG. 3. (Color online) (a) Measured tunneling spectrum of film I (solid curve). (b) Average tunneling spectrum of film C (solid curve) at $T=50 \mathrm{mK}$, together with the normal-state tunneling spectrum at $T=1.4 \mathrm{~K}$ [dashed (red) curve]. (c) Tunneling spectra of film C at three positions (solid curves) and the average tunneling spectrum (dashed curve). Curves are shifted for clarity (dotted lines give the zero-conductance levels). The normal-state background is removed from these measurements (see text for details). Dash-dotted black curves in (a) and (c) are the tunneling spectra that are expected from the electrodynamics measurements. (d) Histogram of the characteristic energy scale $E_{\mathrm{t}}$ in the local spectra in film $\mathrm{C}$. The Gaussian fit (solid curve) is used to extract the mean value.

it to play a role in the electrodynamic response of the film. Consequently, we have divided all measured spectra by the average normal state tunneling spectrum [dashed (red) curve].

Figure 3(c) shows the resulting tunneling spectra, obtained at three positions, where a small linear background, due to thermal drift in the tip-sample distance, is subtracted. From these curves, it is clear that the local properties of the film vary on the 100-nm length scale. First, the height of the coherence peak varies from point to point. There are locations where a distinct coherence peak is seen (top curves), as well as locations where the coherence peak is absent. In addition, the typical energy scale $E_{\mathrm{t}}$, defined as the crossing point with the normal-state tunneling spectrum, varies from point to point. On average, $E_{\mathrm{t}}=134 \mu \mathrm{eV}$, with a variation of $20 \%$ [shown in Fig. 3(d)]. This variation in energy scale and coherence peak height is of a similar size as observed in experiments in strongly disordered $\mathrm{TiN}^{15}{ }^{15} \mathrm{InO}_{x},{ }^{2}$ and $\mathrm{NbN} .{ }^{19}$ It is consistent with numerical work relating it to homogeneous on-site disorder in competition with superconductivity. ${ }^{20,21}$

Let us now compare the local spectroscopy measurements to the results obtained from the electrodynamics. The dashdotted curves in Fig. 3 show the tunneling spectra that were 
calculated using the values inferred from the electrodynamic response as listed in Table I. The curves are a convolution of the quasiparticle density of states calculated using Eq. (2) with the derivative of the Fermi-Dirac distribution function describing thermal excitations in the normal-metal tip. For the calculation of the curves, there is no fitting parameter, except for a scaling parameter which is proportional to the tunneling conductance of the contact. The effective temperature of the tip is taken to be equal to the bath temperature $(T=1.35 \mathrm{~K})$ in the case of film I and assumed to be $300 \mathrm{mK}$ in the case of film $\mathrm{C}$, based on previous experience with this measurement setup.

In the case of the least-disordered film, I, there is a clear correspondence between the measurements and the predictions from the model: the size and width of the coherence peaks and their distance are accurately reproduced. Note that this confirms that already for relatively clean films, the density of states is severely changed from the BCS case with a singularity at $E=\Delta$ (shown in the inset in Fig. 2), in contrast to what is generally assumed for TiN films. ${ }^{22,23}$ In the case of the mostdisordered superconducting film $\mathrm{C}$, the picture is different. The measured curves are not predicted by the model. First, in most of the spectra, coherence peaks are clearly visible, while they do not follow from the Usadel equation, (2), for the parameters listed in Table I. Second, the calculated curve is much smoother: even on positions where the coherence peaks are absent [bottom curve in Fig. 3(c)], there is still a clear onset of a gap, which is not reproduced by the model.

These differences may be attributed to the tunneling measurement method. It was recently suggested ${ }^{24}$ that the metallic tip not only is a passive probe of the tunneling density of states, but also may influence the electron-electron interaction processes in the highly disordered films themselves, thereby yielding a stronger superconducting state than observed in the electrodynamics measurements. Although we cannot rule out such an influence on a quantitative level, we believe that this cannot explain the observed spatial variation in the local superconducting density of states.

In our view, the main result of the STS measurements are the local variations of the superconducting state. In contrast, for the electrodynamic properties we apply a model which assumes uniform properties, with an effective pair-breaking parameter. Since the wavelength of the microwaves used in our experiment is large enough to probe an average over the local landscape, an effective uniform model might still be appropriate. However, there is no a priori reason why this is represented by an effective pair breaker. In fact, the averaged tunnel spectrum, shown in Fig. 3(c), reveals a stronger superconducting state than is predicted by the electrodynamics measurements. A proper theoretical description of the microwave response will need to start with an inhomogeneous superconducting state. We are not aware of such a theory.

Finally, in analyzing the density of states as observed in our STS measurements of film $\mathrm{C}$, we appear to observe an appreciable number of subgap states. Also, in this case we are reluctant to apply a model which assumes a uniform superconductor with subgap states. For a nonuniform superconducting state the lateral proximity effect will contribute to the locally measured density of states, which may appear as subgap states. ${ }^{25}$ Moreover, the influence of the electronelectron interactions, leading to the normal-state tunneling density of states, on the superconducting state are not clear. A thorough understanding of the local tunneling spectra in an inhomogeneous superconducting medium is needed before one can infer a macroscopic quantity such as the response to microwaves.

In summary, we have clearly shown that the microwave electrodynamics of strongly disordered superconductors into a regime up to $k_{\mathrm{F}} l=0.82$ can be measured using a hybrid sample design. We have also shown that even in this regime we can describe the electrodynamic response with a model which contains a disorder-dependent effective pair breaker. For the least disordered film $\left(k_{\mathrm{F}} l=8.6\right)$, these results are consistent with the local tunneling spectra. For the most disordered film, however, a discrepancy is found, which signals the breakdown of applicability of a model based on averaged properties, due to the emergent electronic inhomogeneity.

We thank Misha Skvortsov, Benjamin Sacépé, Yuli Nazarov, and Jochem Baselmans for useful discussions, Frans Tichelaar for performing the TEM measurements, and Akira Endo and David Thoen for assistance in resonator design and process development. This research was funded by the Dutch Foundation for Research of Matter (FOM) and by the Agence Nationale de la Recherche under the programs POSTIT and QuDEC. This work was supported as part of a collaborative project, SPACEKIDS, funded via Grant No. 313320 provided by the European Commission under Theme SPA.2012.2.2-01 of Framework Programme 7. T.M.K. acknowledges financial support from the Ministry of Science and Education of Russia under Contract No. 14.B25.31.0007. E.F.C.D. was financially supported by the CEA-Eurotalents program. *p.c.j.j.coumou@tudelft.nl

†eduard.driessen@cea.fr

${ }^{1}$ V. F. Gantmakher and V. T. Dolgopolov, Phys. Usp. 53, 1 (2010).

${ }^{2}$ B. Sacépé, T. Dubouchet, C. Chapelier, M. Sanquer, M. Ovadia, D. Shahar, M. V. Feigel'man, and L. B. Ioffe, Nat. Phys. 6, 1 (2011).

${ }^{3}$ E. F. C. Driessen, P. C. J. J. Coumou, R. R. Tromp, P. J. de Visser, and T. M. Klapwijk, Phys. Rev. Lett. 109, 107003 (2012).

${ }^{4}$ M. V. Feigel'man and M. A. Skvortsov, Phys. Rev. Lett. 109, 147002 (2012).

${ }^{5}$ P. Diener, H. G. LeDuc, S. J. C. Yates, Y. J. Y. Lankwarden, and J. J. A. Baselmans, J. Low Temp. Phys. 167, 305 (2012).
${ }^{6}$ N. N. Iossad, A. V. Mijiritskii, V. V. Roddatis, N. M. van der Pers, B. D. Jackson, J. R. Gao, S. N. Polyakov, P. N. Dmitriev, and T. M. Klapwijk, J. Appl. Phys. 88, 5756 (2000).

${ }^{7}$ In the case of film $\mathrm{C}$, the fabrication process was slightly different, resulting in the TiN layer still being present beneath the NbTiN layer.

${ }^{8}$ P. C. J. J. Coumou, M. R. Zuiddam, E. F. C. Driessen, P. J. de Visser, J. J. A. Baselmans, and T. M. Klapwijk, IEEE T Appl. Superconduct. 23, 7500404 (2013).

${ }^{9}$ J. Zmuidzinas, Annu. Rev. Cond. Mat. Phys. 3, 169 (2012). 
${ }^{10}$ See Supplemental Material at http://link.aps.org/supplemental/ 10.1103/PhysRevB.88.180505 for detailed dimensions of the sample design and estimation of the thickness of the various films.

${ }^{11}$ The volume of the reaction chamber was reduced, which presumably reduced the impurity concentration in the films.

${ }^{12}$ This film is labeled $E$ in Ref. 3.

${ }^{13}$ T. I. Baturina, A. Bilušić, A. Y. Mironov, V. M. Vinokur, M. R. Baklanov, and C. Strunk, Physica C: Superconduct. 468, 316 (2008).

${ }^{14}$ M. S. Khalil, M. J. A. Stoutimore, F. C. Wellstood, and K. D. Osborn, J. Appl. Phys. 111, 054510 (2012).

${ }^{15}$ B. Sacépé, C. Chapelier, T. I. Baturina, V. M. Vinokur, M. R. Baklanov, and M. Sanquer, Phys. Rev. Lett. 101, 157006 (2008).

${ }^{16}$ S. Nam, Phys. Rev. 156, 470 (1967).

${ }^{17}$ B. L. Altshuler and A. G. Aronov, Electron-Electron Interaction in Disordered Conductors (Elsevier, Amsterdam, 1985).
${ }^{18}$ F. Pierre, H. Pothier, P. Joyez, N. O. Birge, D. Esteve, and M. H. Devoret, Phys. Rev. Lett. 86, 1590 (2001).

${ }^{19}$ G. Lemarié, A. Kamlapure, D. Bucheli, L. Benfatto, J. Lorenzana, G. Seibold, S. C. Ganguli, P. Raychaudhuri, and C. Castellani, Phys. Rev. B 87, 184509 (2013).

${ }^{20}$ A. Ghosal, M. Randeria, and N. Trivedi, Phys. Rev. Lett. 81, 3940 (1998).

${ }^{21}$ A. Ghosal, M. Randeria, and N. Trivedi, Phys. Rev. B 65, 014501 (2001).

${ }^{22}$ P. Diener, H. Schellevis, and J. J. A. Baselmans, Appl. Phys. Lett. 101, 252601 (2012).

${ }^{23}$ U. S. Pracht, M. Scheffler, M. Dressel, D. F. Kalok, C. Strunk, and T. I. Baturina, Phys. Rev. B 86, 184503 (2012).

${ }^{24}$ D. Sherman, B. Gorshunov, S. Poran, J. Jesudasan, P. Raychaudhuri, N. Trivedi, M. Dressel, and A. Frydman, arXiv:1304.7087v1.

${ }^{25}$ S. Guéron, H. Pothier, N. O. Birge, D. Esteve, and M. H. Devoret, Phys. Rev. Lett. 77, 3025 (1996) 